\title{
DUALS OF $K$-OPERATOR FRAMES IN HILBERT SPACES
}

\author{
CHANDER SHEKHAR ${ }^{\dagger}$ AND S.K. KAUSHIK \\ Date of Receiving : $\quad$ : 12.04 .2017 \\ Date of Revision : $\quad 11.11 .2017$ \\ Date of Acceptance : 10.01 .2018
}

\begin{abstract}
We characterize an operator which preserve $K$-operator frame and generate $K$-operator frames from old $K$-operator frames. Also, $K$-dual of a $K$ operator frame is defined and gave some of its characterizations.
\end{abstract}

\section{Introduction}

Frames are the redundant system which gives basis like expansion of element of underlying spaces. In 1952 Duffin and Schaeffer [6] introduced frame which gives bases like expansion of a vector in Hilbert spaces. Recall that a countable sequence $\left\{f_{k}\right\}_{k \in \mathbb{N}} \subset \mathcal{H}$ is called frame if there exists positive constants $A$ and $B$ such that

$$
A\|f\|^{2} \leq \sum_{i, k \in \mathbb{N}} \mid\left\langle f,\left.f_{k}\right|^{2} \leq B\|f\|^{2}, f \in \mathcal{H} .\right.
$$

Since then, various generalizations viz. $g$-frames [13], Fusion frames $[1,3]$, operator valued frames [8] of frames have been obtained. Găvruţa [9] gave the generalization of frames in the form of $K$-frame which has been further generalized by [4] and [12] in the setting of operators. $K$-frames were further studied in $[10,11]$. In this paper, we characterize operator which preserve $K$-operator frames and generates new frames from the old ones. We have also obtained some results related to sum of $K$-operator frames. Further, we have defined the notion of $K$-dual of $K$-operator frames. Finally, necessary and sufficient conditions in the form of various results for $K$-dual of $K$-operator frames have been given. This paper is organized as follows: In section 2, we give some basic tools which is needed throughout the paper. In section 3 , we prove image of $K$-operator frame under an operator is a $K$-operator frame. Also, we prove that sum of $K$-operator frame and image of $K$-operator frame under an operator is again a $K$-operator frame. In section 4 , we define $K$-dual of $K$-operator frame.

2010 Mathematics Subject Classification. 42C15, $42 \mathrm{C} 38$.

Key words and phrases. Frames, $K$ operator frames, Bessel sequences.

Communicated by. Laura Gavruta

${ }^{\dagger}$ Corresponding author. 